\title{
First Order Perturbation-based Stochastic Homogenization Analysis for Short Fiber Reinforced Composite Materials*
}

\author{
Sei-ichiro SAKATA**, Fumihiro ASHIDA** and Ryuta TANAKA*** \\ ${ }^{* *}$ Shimane University, \\ 1060 Nishikawatsu-cho, Matsue, Shimane, Japan \\ E-mail: sakata@ecs.shimane-u.ac.jp \\ ${ }^{* * *}$ Graduate School of Shimane University, \\ 1060 Nishikawatsu-cho, Matsue, Shimane, Japan
}

\begin{abstract}
This paper describes stochastic homogenization analysis of a uni-directionally aligned short fiber reinforced composite material. In case of a short fiber reinforced composite material, length or orientation of the short fiber will be a random variable in addition to elastic properties of component materials, volume fraction or cross-sectional shape of fiber. Especially, a stochastic homogenization problem considering the length or fiber orientation variation is analyzed in this paper. For the purpose of this analysis, the first-order perturbation-based stochastic homogenization method is employed. Since the perturbation term with respect to the length or the orientation variation cannot be explicitly obtained in using the homogenization method, a finite differential technique is used for approximation of the perturbation term. From the numerical results, validity, effectiveness and problem of the perturbation-based analysis are discussed. Also, influence of the length variation on a stochastic characteristic of a homogenized elastic property of a short fiber reinforced composite material is discussed.
\end{abstract}

Key words: Stochastic Homogenization Analysis, Perturbation, Homogenization Method, Short Fiber Reinforced Composites

\section{Introduction}

Since a microscopic random variation will have an influence on a homogenized material property of a composite material, a stochastic homogenization problem has been analyzed in recent. Kaminski ${ }^{(1)-(3)}$, Ostojya, ${ }^{(4)}$ Sakata ${ }^{(5)-(7)}$ or $\mathrm{Xu}^{(8)}$ reported several methods for the stochastic homogenization analysis. This kind of a problem has been noticed for other objectives, for instance, Lombardo ${ }^{(9)}$ reported a stochastic modeling for a masonry structure.

For a composite material, a unidirectional continuous fiber reinforced composite material is sometimes selected as a target of the analysis, and in this case a two dimensional problem is sometimes assumed. It may be effective for simplifying the problem, but a three dimensional analysis should be performed for a more complex problem observed in several kinds of composite materials. For instance, a short fiber reinforced composite material has a three dimensional microstructure, and the stochastic homogenization problem for the short fiber reinforced composites will be more complex than that of a unidirectional fiber

*Received 1 Oct., 2009 (No. 09-0554) [DOI: 10.1299/jmmp.4.446]

Copyright () 2010 by JSME 
reinforced composites even if an assumption as the short fiber is uni-directionally aligned can be acceptable.

From this viewpoint, a stochastic homogenization analysis of a short fiber reinforced composite material is attempted in this paper. About a short fiber reinforced composite material, length or orientation of fiber will be a random variable in addition to elastic properties of component materials, volume fraction or cross-sectional shape of fiber. Especially, a stochastic homogenization problem considering the length or fiber orientation variation is analyzed in this paper. For the purpose of the analysis, the first-order perturbation-based stochastic homogenization method is employed. Since the perturbation term with respect to the length or the orientation cannot be explicitly obtained in using the finite element-based analysis, a finite differential technique is used for approximation of the perturbation term.

At first, outline of the stochastic homogenization analysis is introduced. Next, the problem setting and numerical results are described. With the numerical results, applicability of the perturbation-based approach to the problem and influence of the microscopic uncertainty on the stochastic characteristics of the homogenized elastic properties are discussed.

\section{Analysis method}

\subsection{Homogenization Method}

A homogenized elastic tensor of a composite material can be computed using the homogenization method ${ }^{(10)(11)}$ as

$$
\boldsymbol{E}^{H}=\frac{1}{|\boldsymbol{Y}|} \int_{Y} \boldsymbol{E}\left(\boldsymbol{I}-\frac{\partial \chi}{\partial \boldsymbol{y}}\right) \mathrm{d} \boldsymbol{y}
$$

where $\boldsymbol{E}^{H}$ is a homogenized elastic tensor, $\boldsymbol{I}$ is a unit tensor, $|\boldsymbol{Y}|$ is volume of a unit cell, $\boldsymbol{E}$ is a elastic tensor of a microstructure, $\boldsymbol{y}$ is a variable with respect to microscopic coordinates and $\chi$ is a characteristic displacement. $\chi$ is obtained as a solution of the following characteristic equation.

$$
\int_{Y} \frac{\partial}{\partial \boldsymbol{y}} \boldsymbol{E} \frac{\partial \chi}{\partial \boldsymbol{y}} \mathrm{d} \boldsymbol{y}=\int_{Y} \frac{\partial}{\partial \boldsymbol{y}} \boldsymbol{E} \mathrm{d} \boldsymbol{y}
$$

Equation (2.2) can be discretized using the finite element method as

$$
[K][\chi]=[\boldsymbol{F}]
$$

where $[K]$ is a coefficient matrix of the characteristic equation for a unit cell, $[\boldsymbol{F}]$ shows a set of force vectors caused by inhomogeneity in material properties of a microstructure.

\subsection{First-Order Perturbation-based Stochastic Homogenization Method ${ }^{(2),(5),(12)}$}

If a random variation of a microscopic material property is taken into account, each term of equation (2.3) will have a random variation, then it can be rewritten as

$$
\left[K^{*}\right]\left[\chi^{*}\right]=\left[\boldsymbol{F}^{*}\right]
$$

where the superscript "*” shows an observed value of a probabilistic response function.

With using the first order perturbation theory, each term of Eq. (2.4) can be expressed by the following asymptotic expansion forms

$$
\begin{aligned}
& {\left[K^{*}\right] \approx\left[K^{0}\right]+\left[K^{1}\right] \alpha} \\
& {\left[\chi^{*}\right] \approx\left[\chi^{0}\right]+\left[\chi^{1}\right] \alpha} \\
& {\left[\boldsymbol{F}^{*}\right] \approx\left[\boldsymbol{F}^{0}\right]+\left[\boldsymbol{F}^{1}\right] \alpha}
\end{aligned}
$$

where the subscript " 0 " or " 1 " shows the 0 th or first order perturbation term and $\alpha$ is a random variable. Comparing with the coefficients with respect to $\alpha$ and the constant 
term, we can obtain a simultaneous equation as

$$
\left.\begin{array}{c}
{\left[K^{0}\right]\left\{\chi^{0}\right\}=\left[F^{0}\right]} \\
\left(\left[K^{1}\right]\left\{\chi^{0}\right\}+\left[K^{0}\right]\left\{\chi^{1}\right\}\right)=\left[F^{1}\right]
\end{array}\right\}
$$

Also, an asymptotic form of a homogenized elastic tensor can be expressed as

$$
\begin{aligned}
{\left[E^{H^{*}}\right] } & \approx\left[E^{H 0}\right]+\left[E^{H 1}\right] \alpha \\
& =\frac{1}{|\boldsymbol{Y}|} \int_{Y}\left(\left[E^{0}\right]+\left[E^{1}\right] \alpha\right) \mathrm{d} y \\
& -\frac{1}{|\boldsymbol{Y}|} \int_{Y}\left(\left[E^{0}\right]+\left[E^{1}\right] \alpha\right)\left(\left[B^{0}\right]+\left[B^{1}\right] \alpha\right)\left(\left\{\chi^{0}\right\}+\left\{\chi^{1}\right\} \alpha\right) \mathrm{d} y
\end{aligned}
$$

where $[B]$ is a displacement-strain matrix.

Comparing with the each coefficient, each perturbation term of the homogenized elastic tensor can be obtained using the following equations.

$$
\left.\begin{array}{l}
{\left[E^{H 0}\right]=\frac{1}{|\boldsymbol{Y}|} \int_{Y}\left\langle\left[E^{0}\right]-\left[E^{0}\right]\left[B^{0}\right]\left\{\chi^{0}\right\}\right) \mathrm{d} y} \\
{\left[E^{H 1}\right]=\frac{1}{|\boldsymbol{Y}|} \int_{Y}\left\langle\left[E^{1}\right]-\left(\left[E^{0}\right]\left[B^{0}\right]\left\{\chi^{1}\right\}+\left[E^{0}\right]\left[B^{1}\right]\left\{\chi^{0}\right\}+\left[E^{1}\right]\left[B^{0}\right]\left\{\chi^{0}\right\}\right)\right\rangle \mathrm{d} y}
\end{array}\right\}
$$

In case of an orthotropic material, a stochastic form of homogenized equivalent elastic constants can be computed as ${ }^{(5)}$

$$
\left.\begin{array}{l}
E_{x}^{H^{*}}=\frac{1}{C_{11}{ }^{H^{*}}}, \quad E_{y}{ }^{H^{*}}=\frac{1}{C_{22}{ }^{H^{*}}}, \quad E_{z}^{H^{*}}=\frac{1}{C_{33}{ }^{H^{*}}} \\
v_{y z}{ }^{H^{*}}=-E_{y}{ }^{H^{*}} C_{23}{ }^{H^{*}}, \quad v_{z x}{ }^{H^{*}}=-E_{z}{ }^{H^{*}} C_{31}{ }^{H^{*}}, \quad v_{x y}{ }^{H^{*}}=-E_{x}{ }^{H^{*}} C_{12}{ }^{H^{*}} \\
G_{y z}{ }^{H^{*}}=\frac{1}{C_{44}{ }^{H^{*}}}, \quad G_{z x}{ }^{H^{*}}=\frac{1}{C_{55}{ }^{H^{*}}}, \quad G_{x y}{ }^{H^{*}}=\frac{1}{C_{66}{ }^{H^{*}}}
\end{array}\right\}
$$

where $\boldsymbol{C}^{H}$ is a homogenized compliance matrix, which can be computed as the inverse of the homogenized elastic tensor $\boldsymbol{E}^{H} . \boldsymbol{C}^{H^{*}}$ can be also approximated with the asymptotic expansion as Eq.(2.12), then the first order perturbation term of the equivalent elastic constant can be computed as Eq.(2.13)

$$
\begin{gathered}
C_{i j}{ }^{{ }^{*}} \approx\left\{\begin{array}{l}
\frac{Q^{0}{ }_{i j}+Q^{1}{ }_{i j} \alpha+\cdots}{R^{0}+R^{1} \alpha+\cdots} \quad: i, j=1 \sim 3 \\
\frac{1}{E_{i j}{ }^{*}} \quad: i, j=(4,4),(5,5),(6,6)
\end{array}\right. \\
E_{x}{ }^{H 1}=\frac{R^{1} Q_{11}{ }^{0}-Q_{11}{ }^{1} R^{0}}{Q_{11}{ }^{0}}, \quad v_{x y}{ }^{H 1}=-\left(C_{12}{ }^{H 1} E_{x}{ }^{H 0}+C_{12}{ }^{H 0} E_{x}{ }^{H 1}\right)
\end{gathered}
$$

where $Q$ and $R$ are constants computed from the perturbation term of the homogenized elastic tensor.

Using a result of the perturbation-based analysis, the stochastic characteristics such as the expectation and variance of a homogenized elastic tensor or homogenized equivalent elastic constants can be estimated using the first order second moment method ${ }^{(13)}$ as

$$
\left.\begin{array}{l}
\mathrm{E}\left[E^{H}\right]=E^{H 0} \\
\operatorname{Var}\left[E^{H}\right]=\sum_{i} \sum_{j} E_{i}^{H 1} E_{j}^{H 1} \operatorname{cov}\left[\alpha_{i}, \alpha_{j}\right]
\end{array}\right\}
$$

where $\operatorname{cov}\left[\alpha_{i}, \alpha_{j}\right]$ is the covariance of $\alpha_{i}$ and $\alpha_{j}$.

2.3 Perturbation-based analysis for a short fiber reinforced composite material

In case of a short fiber reinforced composite material, some geometrical parameters such 
as the length or orientation of fiber will be taken into account as a random variable in addition to the elastic properties, volume fraction or cross-sectional shape of fiber, which is treated as a random variable in case of a unidirectional fiber reinforced composite material.

In this paper, as a first step of this study, a uni-directionally aligned short fiber reinforced composite material, which is illustrated in Fig.1 (a), is assumed ${ }^{(14)}$ for simplification. Shape of a short fiber is assumed as ellipsoidal as shown in Fig. 1(b). The length or orientation of the fiber can be randomly varied as Fig.1(c).

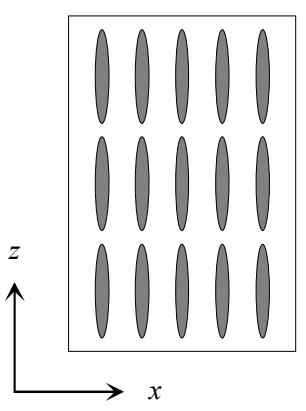

(a)short fiber reinforced composite

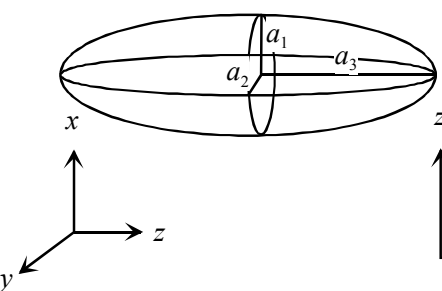

(b)shape of a short fiber (c)random variation in length or orientation

Fig 1. Scheme of a unidirectionally aligned short fiber reinforced composite material

For evaluation of a stochastic characteristic of a homogenized elastic property of the composite material caused by a random variation in an elastic property of a component material, the perturbation technique described in the previous section can be usable. However, in case of a geometrical variation, an additional numerical effort will be needed for the stochastic homogenization analysis. For a geometrical random variation, each perturbation term of the homogenized elastic tensor can be expressed as

$$
\left.\begin{array}{l}
{\left[E^{H 0}\right]=\frac{1}{|\boldsymbol{Y}|} \int_{Y}[E]\left([I]-\left[B^{0}\right]\left[\chi^{0}\right]\right) \mathrm{d} y} \\
{\left[E^{H 1}\right]=\frac{1}{|\boldsymbol{Y}|} \int_{Y}\left\{-[E]\left(\left[B^{1}\right]\left[\chi^{0}\right]+\left[B^{0}\right]\left[\chi^{1}\right]\right)\right\} \mathrm{d} y}
\end{array}\right\}
$$

From this equation, it is recognized that the first order perturbation term of the displacement-strain matrix $\left[B^{1}\right]$ is needed. If each nodal coordinate is a random variable, $\left[B^{1}\right]$ can be obtained analytically. However, for a typical geometrical random variation such as the length or orientation variation, it cannot be explicitly expressed as a function of a random variable in case of using the finite element method based approach. From this reason, the first order perturbation term of the homogenized elastic tensor with respect to the length or orientation variation is approximated using the finite differential method. The approximated first order perturbation term of the homogenized elastic tensor can be written as

$$
\left[E^{H 1}\right] \approx \frac{1}{|\boldsymbol{Y}|} \int_{Y}\left\{\frac{-[E]}{\mathrm{d} \alpha}\left([\Delta B]\left[\chi^{0}\right]+\left[B^{0}\right][\Delta \chi]\right)\right\} \mathrm{d} y=\frac{\left[\Delta E^{H}\right]}{\mathrm{d} \alpha}
$$

For the fiber length variation or fiber orientation variation, the perturbation term can be computed as

$$
\left[E^{H 1}\right]_{l} \approx \frac{\mathrm{d} l}{\mathrm{~d} \alpha} \frac{\left[\Delta_{l} E^{H}\right]}{\mathrm{d} l},\left[E^{H 1}\right]_{\theta} \approx \frac{\mathrm{d} \theta}{\mathrm{d} \alpha} \frac{\left[\Delta_{\theta} E^{H}\right]}{\mathrm{d} \theta}
$$

$\left[\Delta E^{H}\right]$ can be computed from a forward, backward or central difference. 


\subsection{Stochastic Analysis using the Monte-Carlo simulation}

For accuracy evaluation of the perturbation-based approach, the stochastic characteristics of the homogenized equivalent elastic constants with respect to the microscopic random variation are also computed using the Monte-Carlo simulation.

From the Monte-Carlo simulation, the stochastic characteristics such as the expectation or variance of the homogenized elastic properties can be computed as

$$
\begin{gathered}
\mathrm{E}\left[\boldsymbol{E}^{H^{*}}\right] \approx \frac{1}{N} \sum_{i} \boldsymbol{E}^{H^{*}{ }_{i}} \\
\operatorname{Var}\left[\boldsymbol{E}^{H^{*}}\right] \approx \frac{1}{N} \sum_{i}\left(\boldsymbol{E}^{H^{*}}{ }_{i}-\mathrm{E}\left[\boldsymbol{E}^{H^{*}}\right]\right)^{2}
\end{gathered}
$$

where $N$ is the number of trials. Also the coefficient of variance (CV) can be computed as

$$
\mathrm{CV}\left[\boldsymbol{E}^{H^{*}}\right]=\frac{\sqrt{\operatorname{Var}\left[\boldsymbol{E}^{E I^{*}}\right]}}{\mathrm{E}\left[\boldsymbol{E}^{E I^{*}}\right]}
$$

The Box-Mullar randomization ${ }^{(15)}$ is used for generating a set of normal distributed random values for the Monte-Carlo simulation. In this paper, $10^{4}$ trials are performed for the Monte-Carlo simulation.

\section{Problem Settings}

In this paper, the stochastic characteristics of a homogenized equivalent elastic constant of a unidirectionally aligned short fiber reinforced composite caused by a random variation of a material property or geometry of a microstructure are estimated. Four elastic properties as Young's modulus of the fiber $\left(E_{f}\right)$ and resin $\left(E_{m}\right)$, and Poisson's ratios of the fiber $\left(v_{f}\right)$ and resin $\left(v_{m}\right)$ are taken into account as a random variables related to a material property. Also, length $(l)$ and orientation $(\theta)$ of fiber are taken into account as geometrical random variables. The expected values of the elastic properties on fiber and matrix are listed in Table 1. The properties of fiber and matrix are employed correspond to E-glass and Epoxy resin. In this case, the expected value of volume fraction of fiber $\left(V_{f}\right)$ is 0.27 .

It is assumed that the random variables are independently distributed according to the normal distribution. Those observed values can be expressed as

$$
X^{*}=X^{0}(1+\alpha)
$$

and stochastic characteristics of a random variable $\alpha$ are assumed as

$$
\mathrm{E}[\alpha]=0, \operatorname{Var}[\alpha]=\sigma^{2}
$$

where $\mathrm{E}[\alpha]$ is an expected value and $\operatorname{Var}[\alpha]$ is variance of a stochastic variable $\alpha$. In this case, it is assumed that $\sigma=0.05$.

Table 1 Expected values of elastic properties for fiber and matrix

\begin{tabular}{|c|c|c|}
\hline & Fiber ( E-glass ) & Matrix ( Epoxy ) \\
\hline Young's modulus ( GPa ) & 73.0 & 4.5 \\
\hline Poisson's ratio & 0.22 & 0.39 \\
\hline
\end{tabular}




\section{Numerical Results}

4.1 Accuracy of the first order perturbation-based stochastic homogenization method for the length variation

At first, accuracy of the first order perturbation-based stochastic method for the stochastic homogenization analysis of the short fiber reinforced composite material is investigated. Figure 2 shows the relative estimation error between the results of the first order perturbation method and the Monte-Carlo simulation. The expected value of $a_{3}$ is 10.0 in this case. $a_{1}$ and $a_{2}$ are fixed at 1.0. Only the length of fiber is a randomly changed and the orientation is fixed in this case. From Fig.2, it is recognized that the estimation error for the $v_{m}$ variation is larger than the others, and those are less than $1 \%$. This results show that an estimated CV will be reliable for $E_{f}, E_{m}$ and $v_{f}$ variation. The estimated $\mathrm{CV}$ for $v_{m}$ will include a larger error than the others. The estimation errors for the length variation are larger than that for $E_{f}, E_{m}$ and $v_{f}$ variation, but those are less than about $2 \%$. Since a result CV estimation using the Monte-Carlo simulation with $10^{4}$ trials will include about 3-5\% error, these results indicate that the perturbation-method will be usable for the stochastic homogenization analysis. However, it should be also noted the estimation error in $\mathrm{CV}\left[E_{z}\right]$ estimation reaches $6 \%$, and the error may become beyond $10 \%$ in the worst case since the result of MC includes $3-5 \%$ bias. This result shows that the proposed perturbation method should be carefully used for $\mathrm{CV}$ estimation considering a large $v_{m}$ variation.

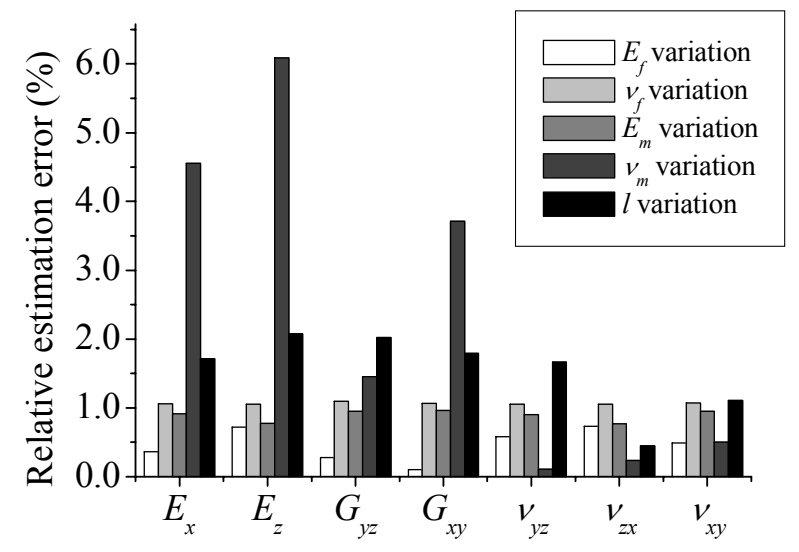

Fig. 2. Estimation error in $\mathrm{CV}$ of the homogenized elastic constants for each material property variation

In order to investigate influence of the fiber length on the estimation accuracy of the perturbation-based technique, the relative estimation errors are computed for different fiber lengths. In this case, the volume fraction is fixed, and length of fiber is changed. As an example, the relationships between $a_{3}$ and the relative error in CV estimation for $E_{f}, v_{m}$ and the length variation are shown in Fig.3.

From this figure, it is recognized that the estimation error for $\mathrm{CV}$ of some kinds of equivalent elastic properties increases according to the fiber length increases, but the maximum estimation error is about $1 \%$ for $a_{3}=20$ for $E_{f}$ variation, or the maximum error is about $115 \%$ for the length variation. The estimation error in CV of the equivalent Young's modulus for fiber direction in case of $v_{m}$ variation is rapidly increases as the fiber length increases, and the maximum value is about $9 \%$ for $a_{3}=20$. From these results, it can be confirmed that the perturbation based technique is usable for the analysis, but it should be noticed that the estimation error in $\mathrm{CV}$ of $E_{z}$ will be large when $a_{3}$ is large in case of $v_{m}$ variation 


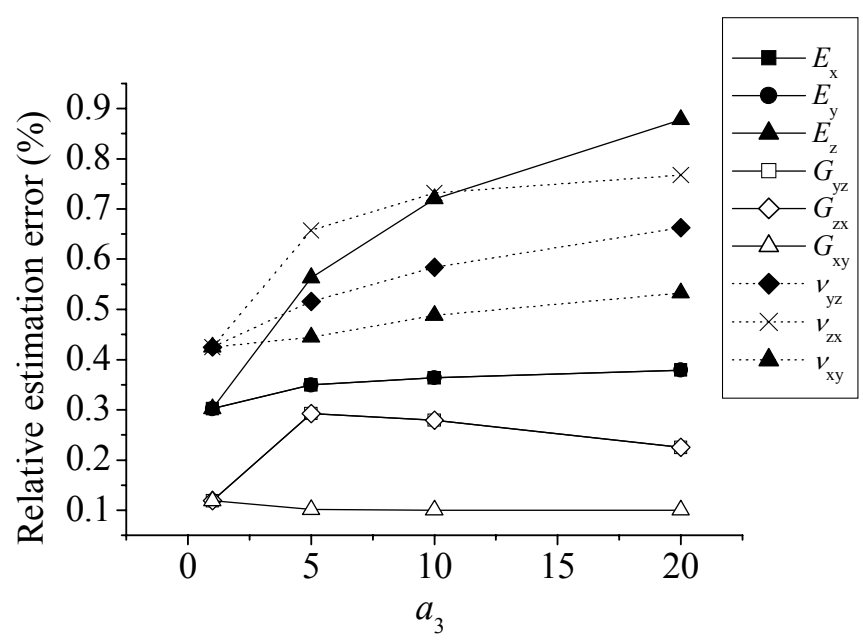

(a) Relative estimation error for each length of fiber in case of $E_{f}$ variation

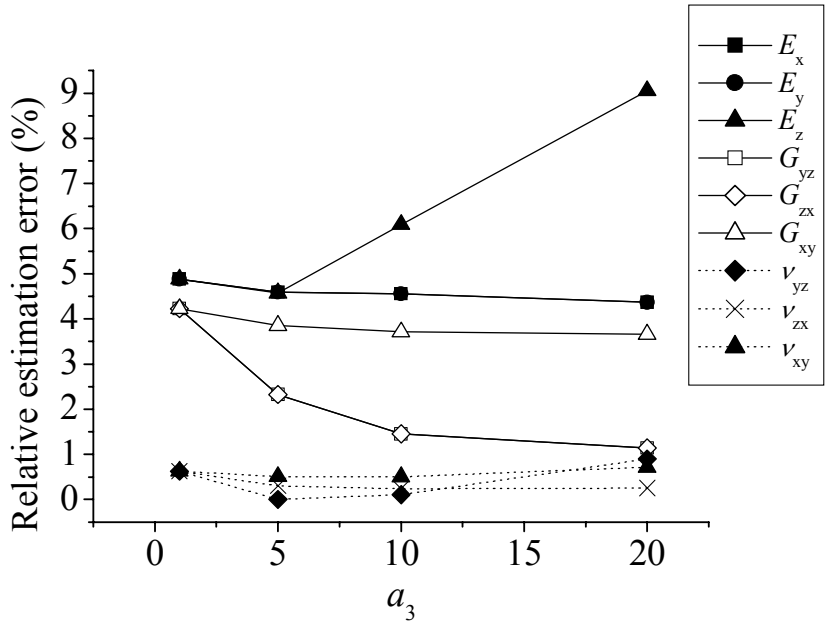

(b) Relative estimation error for each length of fiber in case of $v_{m}$ variation

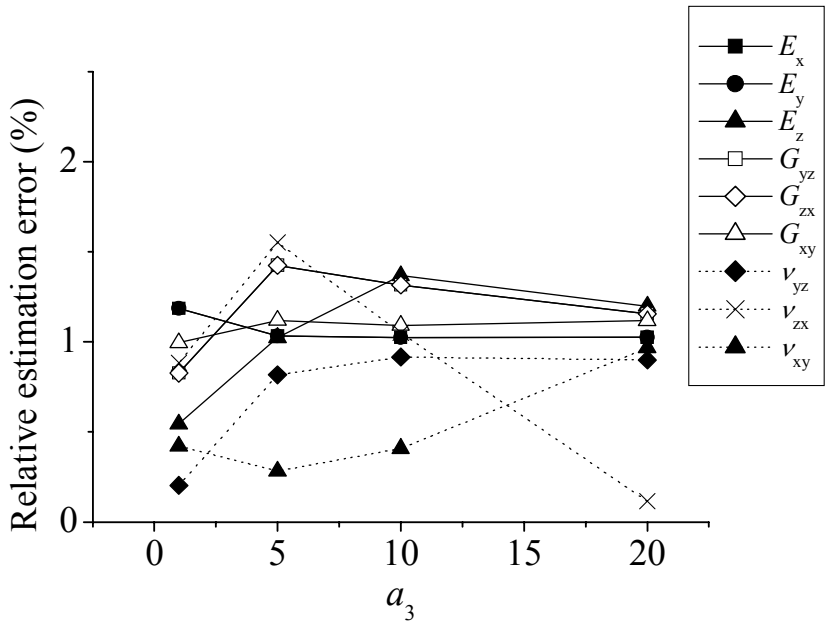

(c) Relative estimation error for each length of fiber in case of the length variation Fig. 3. Estimation error in $\mathrm{CV}$ of the homogenized elastic constants for each material property variation 
4.2 Influence of the fiber length on the stochastic characteristics of a homogenized elastic property

Next, influence of the fiber length on the stochastic characteristics of the homogenized elastic properties is investigated. In particular, relationships between the fiber length and $\mathrm{CV}$ of each homogenized elastic constants caused by a microscopic random variation in material properties of component materials are investigated. The fiber length is changed under assumption on constant volume fraction of fiber. In this section, CV is estimated with using the perturbation-based stochastic homogenization method. Figures 4-7 show the relationship for $E_{f}, E_{m}, v_{f}$ and $v_{m}$ variation. From Fig.4, it is recognized that CV of $E_{z}$ and $v_{y z}$ rapidly increase as the fiber length increases in the case of $E_{f}$ variation. On the other hand, in case of $E_{m}$ variation, $\mathrm{CV}$ of $E_{z}$ decreases as the length increases though $\mathrm{CV}$ of $v_{y z}$ increases. From these results, it is considered that influence of the fiber length will be different according to a kind of random variation.

As for the Poisson's ratio variation, it is recognized that the CV of the homogenized Poisson's ratio for $v_{f}$ variation rapidly increases as the length increases. On the other hand, $\mathrm{CV}$ of $E_{z}$ for $v_{m}$ variation decreases when the length is larger than 5. From this result and Fig.4, it can be considered that the most $\mathrm{CV}$ caused by a material property variation of the fiber will increase as the length increase. Also, it is recognized that the influence of the length on the $\mathrm{CV}$ caused by variation of an elastic property of the matrix will be more complex than that of the fiber; some of them will increase when the length increases, but others, for instance $\mathrm{CV}$ of $E_{z}$ for $E_{m}$ or $v_{m}$ variation, will decrease if the length increases.

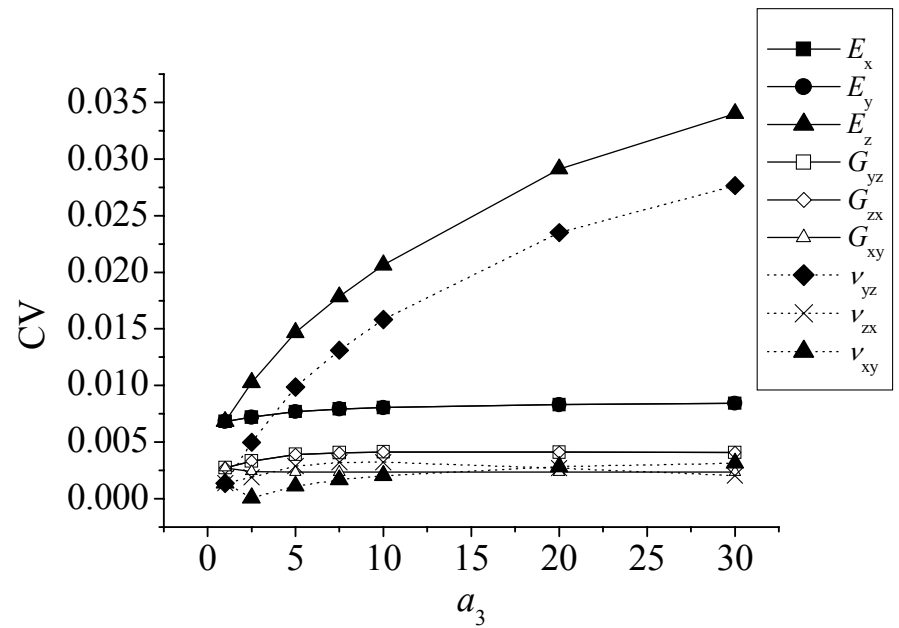

Fig.4 Relationship between the length of fiber and the $\mathrm{CV}$ of the homogenized elastic constants caused by $E_{f}$ variation 


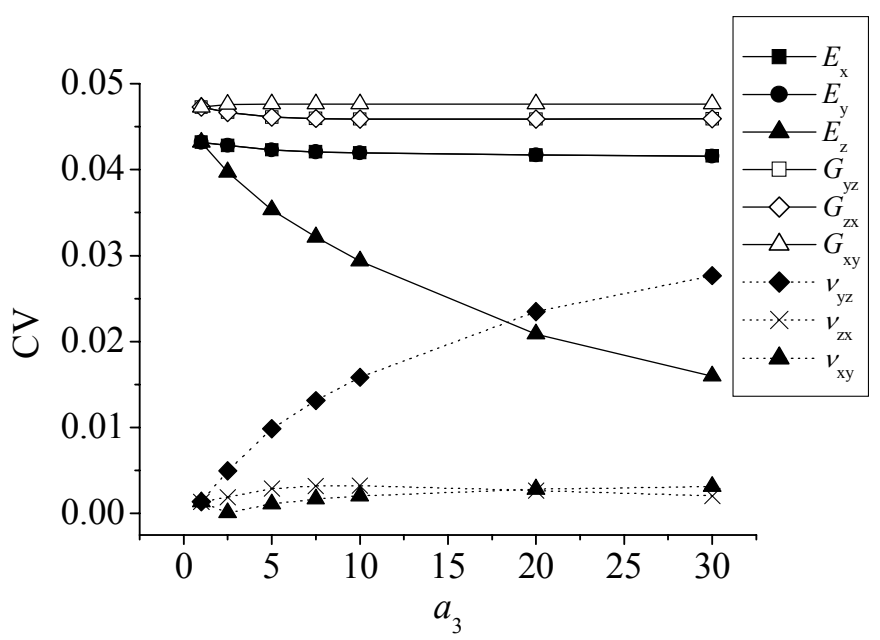

Fig.5 Relationship between the length of fiber and the $\mathrm{CV}$ of the homogenized elastic constants caused by $E_{m}$ variation

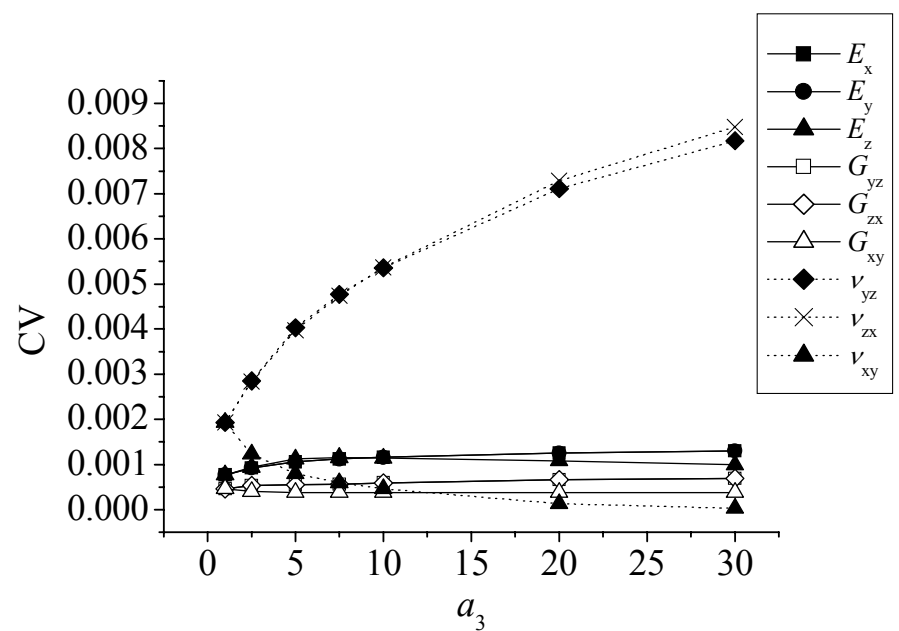

Fig.6 Relationship between the length of fiber and the $\mathrm{CV}$ of the homogenized elastic constants caused by $v_{f}$ variation

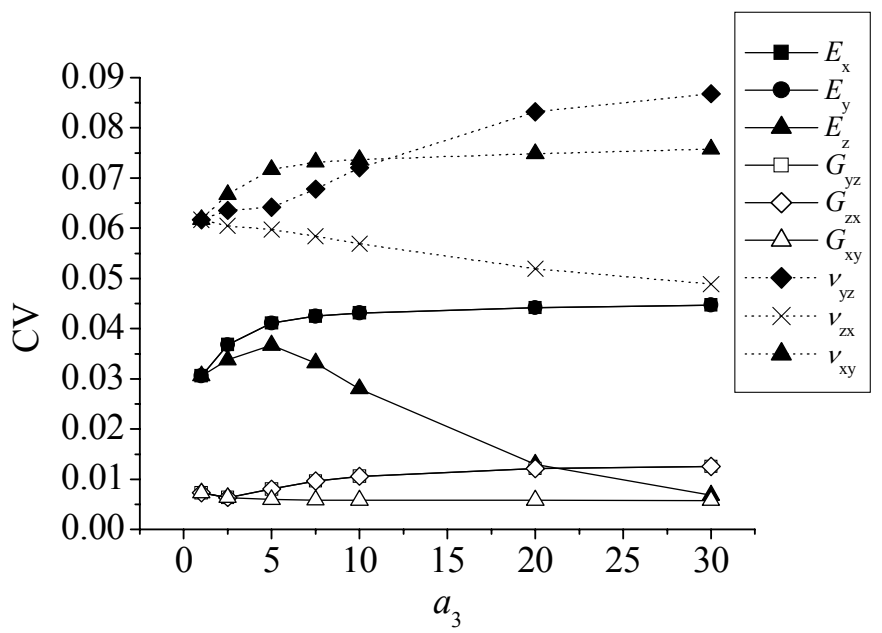

Fig.7 Relationship between the length of fiber and the $\mathrm{CV}$ of the homogenized elastic constants caused by $v_{m}$ variation 
Additionally, influence of the fiber length on the CV caused by the fiber length variation is investigated. A relationship between the CV and the fiber length is shown in Fig.8. In this figure, $\mathrm{CV}$ of the $E_{z}$ and $v_{y z}$ decreases as the length increases when the length is less than 2, then the CV increases when the length is less than 7.5 and then the CV decreases again. The other relationships between $\mathrm{CV}$ and the length are also non-linear, but the influence of the length on the CV caused by the length variation is not larger than that of the elastic property variations. Since the CVs of the homogenized Young's modulus or $v_{y z}$ are similar to or larger than that caused by the material property variation especially in the case that $a_{3}$ is relatively small, the fiber length variation should be taken into account in solving a stochastic homogenization problem in case of a short fiber reinforced composite material.

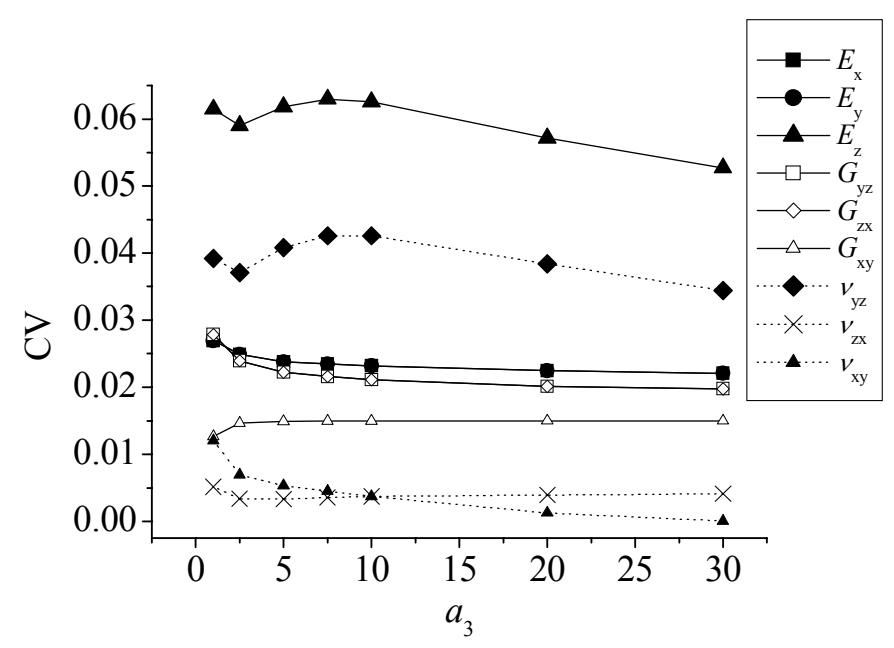

Fig.8 Relationship between the length of fiber

and the $\mathrm{CV}$ of the homogenized elastic constants caused by the length variation

4.3 Influence of a random variation in the length of fiber on the stochastic characteristics of a homogenized elastic property

As an example of a stochastic homogenization analysis of a short fiber reinforced composite material, a stochastic characteristic of the homogenized elastic constants for simultaneous random variations of the all elastic properties of component materials and the fiber length is computed. In order to evaluate influence of the length of fiber and its random variation, relationship between the fiber length and estimated $\mathrm{CV}$ of the homogenized elastic constants are investigated. Figure 9(a) shows a relationship between the CV caused by $E_{f}, E_{m}, v_{f}$ and $v_{m}$ variation. Figure 9(b) shows a relationship between the CV caused by the length variation in addition to those four random variations. From these figures, it is recognized the relationship between the CVs and the length is very similar to each other except to the $\mathrm{CV}$ of $E_{z}$, and the CV of $E_{x}, E_{y}, E_{z}, G_{y z}, G_{z x}$ and $v_{y z}$ with considering the length variation are about $10 \%$ or more larger than the case of considering only elastic property variations. 


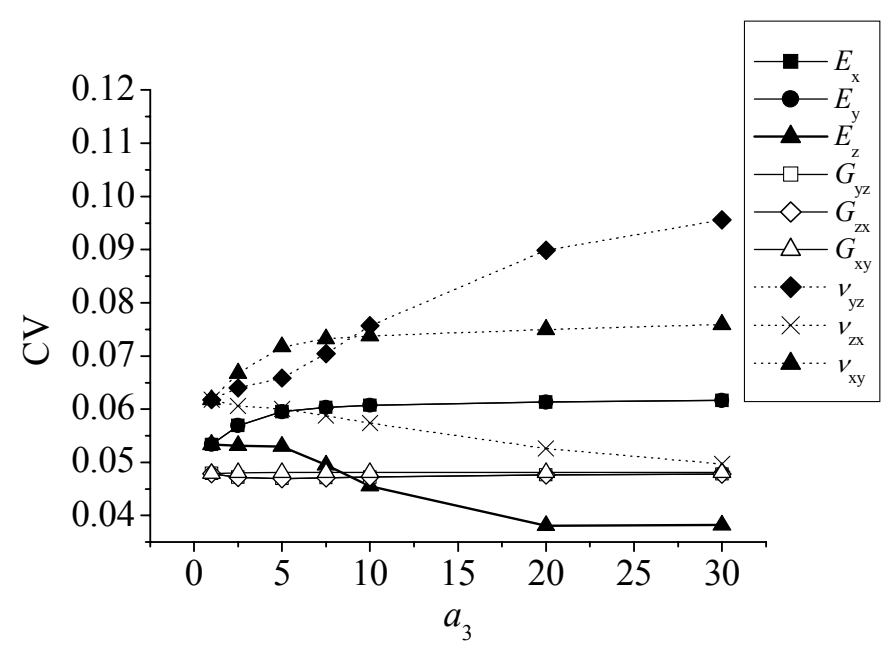

(a) CV for all material properties variations

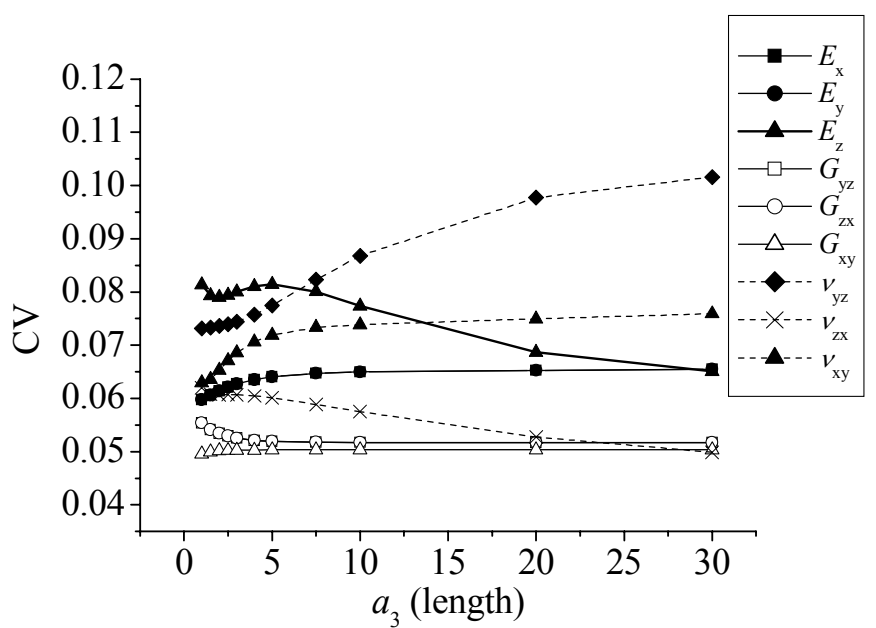

(b) CV for all material properties and length of fiber variations

Fig. $9 \mathrm{CV}$ of the homogenized elastic constants caused by all material properties and the fiber length variations

In order to evaluate influence of the fiber length variation on $\mathrm{CV}$ of the homogenized elastic constants, increasing ratio of the $\mathrm{CV}$ for each fiber length is summarized in Fig.10. $\Delta \mathrm{CV}$ is the increasing ratio, which can be computed as

$$
\Delta \mathrm{CV}=\frac{\mathrm{CV}_{\mathrm{all}}-\mathrm{CV}_{\mathrm{MP}}}{\mathrm{CV}_{\mathrm{MP}}} \times 100
$$

where subscript "all" indicates the $\mathrm{CV}$ caused by $E_{f}, E_{m}, v_{f}, v_{m}$ and the length variation, and subscript "MP" indicates the CV caused by $E_{f}, E_{m}, v_{f}$ and $v_{m}$.

From this figure, it can be recognized that the influence of the fiber length on the $\mathrm{CV}$ of $E_{z}$ is particularly large. $\Delta \mathrm{CV}$ is more than $50 \%$, and it reaches about $80 \%$ when the length is 20. Also, it is recognized that $\Delta \mathrm{CV}$ of $E_{x}, E_{y}, G_{y z}, G_{z x}$ and $v_{y z}$ are more than $10 \%$ when $a_{3}$ is small, and it depends on the length of fiber. This result shows importance of a stochastic homogenization analysis considering the fiber length random variation, and it should be taken into account in analyzing a stochastic characteristic of the homogenized elastic property of a short fiber composite material considering a microscopic random variation in an elastic property of a component material. 


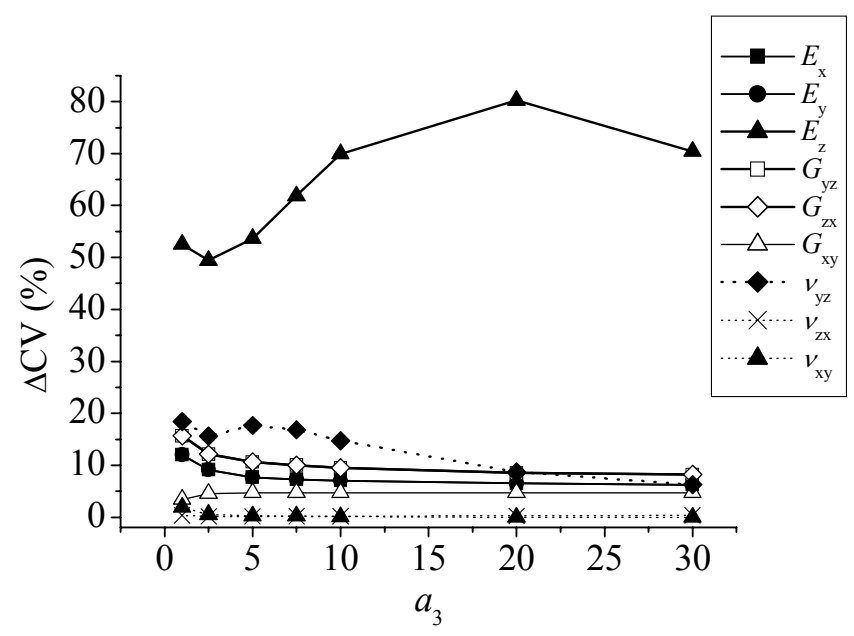

Fig. 10 Influence of the length variation on $\mathrm{CV}$ of the homogenized elastic constants

4.4 Stochastic homogenization analysis considering a random variation in fiber-orientation.

As another geometrical variation, stochastic characteristics of the homogenized elastic constants caused by a random variation of fiber orientation are investigated. In this case, $\sigma=0.04$ is assumed. Table 2 shows the estimated results of $\mathrm{CV}$ for the fiber orientation variation with the perturbation method and the Monte-Carlo simulation.

In this case, three types of the finite differences as forward, backward and central differences are used for computation. From table 2, however, it is recognized that the first order perturbation based approach cannot give an appropriate estimation of the $\mathrm{CV}$ of the homogenized elastic constants for the random variation of the fiber orientation. One of the reasons why the first order perturbation-based approach cannot estimate the CV of the fiber orientation variation is a nonlinear relationship between the equivalent elastic constants and the fiber orientation. As an example, the relationship between some of the equivalent elastic constants and the fiber orientation is illustrated in Fig.11. "Exact" in the figure indicates the response curves obtained from the homogenization analysis with each observed value of the fiber orientation, and "Linear regression" indicates the linear approximation of the curves obtained from the perturbation-based approach using the central finite difference. From this figure, especially in the case of $\mathrm{E}[\theta]=0$, the first order derivative is approximately 0 , and a linear regression using this first order derivative cannot approximate the exact relationship. This fact shows another approach should be developed for estimation of the stochastic characteristics of the homogenized elastic constants for a random variation of fiber orientation.

Table 2 Estimated CVs of homogenized elastic constants for fiber orientation variation

\begin{tabular}{|c|c|c|c|c|c|c|}
\hline & $E^{H}{ }_{x}$ & $E_{z}^{H}$ & $G_{y z}^{H}$ & $G_{x y}^{H}$ & $v_{z x}^{H}$ & $v_{x y}^{H}$ \\
\hline MC & 0.00175 & 0.00759 & 0.0002 & 0.00574 & 0.00582 & 0.0051 \\
\hline FA(forward) & 0.00037 & 0.00165 & 0.00005 & 0.00103 & 0.00118 & 0.00085 \\
\hline FA(backward) & 0.00037 & 0.00165 & 0.00005 & 0.00103 & 0.00118 & 0.00085 \\
\hline FA (central) & 0.00000 & 0.00000 & 0.00000 & 0.00000 & 0.00000 & 0.00000 \\
\hline
\end{tabular}




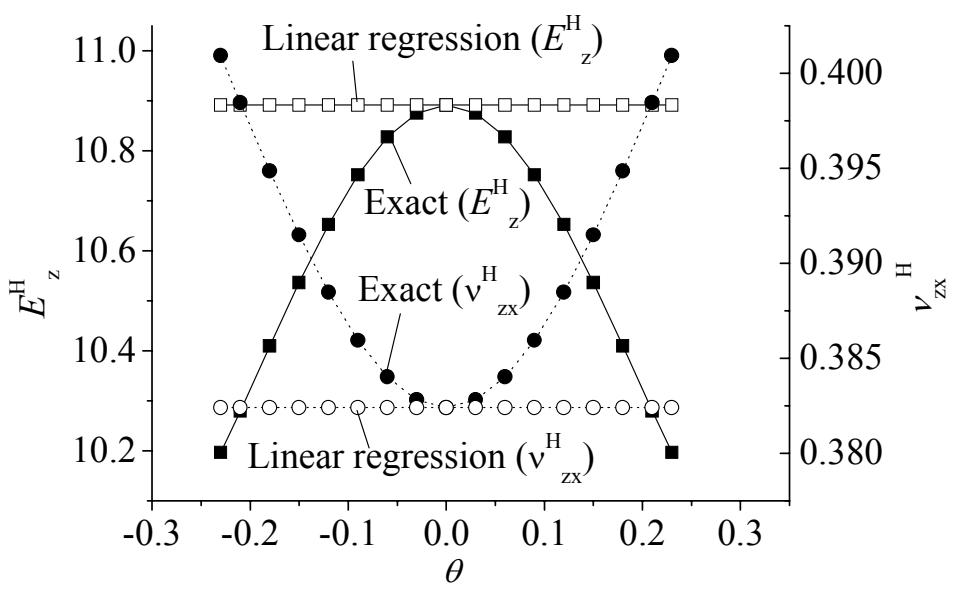

Fig.11 A relationship between the equivalent elastic constants and fiber orientation

\section{Conclusion}

This paper discusses a stochastic homogenization analysis of a unidirectionally aligned short fiber reinforced composite material. Random variables are elastic properties of component materials, length or orientation of fiber. The stochastic properties of the homogenized elastic properties such as the expectation, variance or coefficient of variance are computed with using the first-order perturbation-based stochastic homogenization method. Since it is difficult to compute a perturbation term for the fiber length variation analytically in case of using the finite element method, the finite difference-based approach is employed in this paper.

At first, accuracy of the perturbation-based homogenization method for analysis of the stochastic homogenization problem of a short fiber reinforced composite material and its dependency on the length of fiber are investigated. From the numerical examples, it is confirmed that the first order perturbation-based homogenization method will be usable for the stochastic homogenization analysis considering fiber length variation.

Next, influence of the fiber length on the stochastic characteristics of the homogenized elastic properties is investigated. Also, influence of the fiber length variation on the stochastic characteristics caused by a random variation of elastic properties of component materials is investigated. Dependency and influence of the fiber length and its variation are illustrated with the numerical results. Especially, importance of considering the fiber length variation in stochastic homogenization analysis of a short fiber composite material is confirmed.

Additionally, the perturbation-based homogenization method is applied to stochastic homogenization analysis for a fiber orientation variation. From the numerical results, however, it is recognized that the first order perturbation method is not appropriate to estimate a stochastic characteristic of the homogenized elastic property for the fiber orientation variation. An improved approach should be developed for the analysis in a next step of this study.

\section{Acknowledgement}

The first author is pleased to acknowledge support in part by Grants-in-Aid for Young Scientists (B) (No.21760079) from the Ministry of Education, Culture, Sports Science and Technology. 


\section{References}

[1] Kaminski, M. and Kleiber, M., Stochastic Structural Interface Defects in Fiber Composites, International Journal of Solids and Structures, 33 (20-22), (1996), pp.3035-3056.

[2] Kaminski, M. and Kleiber, M., Perturbation based stochastic finite element method for homogenization of two-phase elastic composites, Computer and Structures, 78, (2000), pp.811-826.

[3] Kaminski, M., Stochastic finite element method homogenization of heat conduction problem in fiber composites, Structural Engineering and Mechanics, 11(4), (2001), pp.373-392.

[4] Ostoja-Starzewski, M., Microstructural Randomness and Scaling in Mechanics of Materials, CRC Press, (2008).

[5] Sakata, S., Ashida, F., Kojima, T. and Zako, M., Three-dimensional stochastic analysis using a perturbation-based homogenization method for elastic properties of composite material considering microscopic uncertainty, International Journal of Solids and Structures, Vol.45, 3/4,(2008), pp.894-907.

[6] Sakata, S., Ashida, F. and Zako, M., Kriging-based Approximate Stochastic Homogenization Analysis for Composite Material, Computer Methods in Applied Mechanics and Engineering, 197(21-24), (2008), pp.1953-1964.

[7] Sakata, S., Ashida, F. and Kojima, T., Stochastic homogenization analysis on elastic properties of fiber reinforced composites using the equivalent inclusion method and perturbation method, International Journal of Solid and Structures, 45, (2008) pp.6553-6565.

[8] Xu, X. F. and Brady, L. G., Computational stochastic homogenization of random media elliptic problems using Fourier Galerkin method, Finite Elements in Analysis and Design, 42, (2005), pp.613-622..

[9] Lombardo, M., Zeman, J., Sejnoha, M. and Falsone, G., Stochastic modeling of chaotic masonry via mesostructural characterization, International Journal for Multiscale Computational Engineering, 7(2), (2009), pp.171-185.

[10] Babu ka, I. , Homogenization approach in engineering. In: J.-L. Lions, R. Glowinski (eds.) Computing Methods in Applied Sciences and Engineering. Lecture Note in Economics and Mathematical Systems 134, Springer: Berlin, ( 1976), pp. 137-153.

[11] Guedes, M. and Kikuchi, N., Preprocessing and postprocessing for materials based on the homogenization method with adaptive finite element methods. Computer Methods in Applied Mechanics and Engineering 83, (1990), pp. 143-198.

[12] Sakata, S., Ashida, F. and Zako, M., Stochastic Response analysis of FRP using the Second-order Perturbation-based Homogenization Method, Journal of Solid Mechanics and Materials Engineering, Vol.2, No.1 (2008), pp.70-81.

[13] S. Nakagiri and T. Hisada, Introduction to the Stochastic Finite Element Method, Baifu-kan. (1985)(in Japanese)

[14] Takao, Y. and Taya, M., Thermal Expansion Coefficients and Thermal Stresses in an Alligned Short Fiber Composite With Application to a Short Carbon Fiber / Alminum, Transactions of the ASME, 52, (1985), pp.806-810..

[15] Press, W. H., Teukolski, S. A., Vetterling, W. T. and Flannery B. P., Numerical Recipes in C ( Japanese Edition), Gijutsu-Hyoron Sha, (1993). (in Japanese) 